\title{
Ethanol Infusion for Alcohol Withdrawal Prophylaxis Does Not Cause Intoxication
}

\author{
Robert Dillard BS, Tanis Welch, PharmD RPh, Senan Abdul-Hamed MD, \\ Jennifer Kesey, MSN, RN, FNP-BC, Sharmila Dissanaike MD, FACS
}

\begin{abstract}
Objective: Alcohol Withdrawal Syndrome (AWS) remains a common problem, especially in trauma and surgical patients. An intravenous ethanol infusion protocol was developed at this institution and previously validated for AWS prophylaxis. One concern with intravenous ethanol has been potential for intoxication and/or side effects. This study was performed on patients receiving AWS prophylaxis with an intravenous ethanol protocol to evaluate for intoxication and the occurrence of any adverse effects.

Methods: We did a retrospective review of all patients in our hospital who received AWS prophylaxis by ethanol infusion between 2008 and 2013. Information collected specific to ethanol infusion included rate of administration, serum ethanol levels, serum sodium level, use of benzodiazepines and anti-emetics, and development of AWS. Intoxication was defined using the Texas limit for blood alcohol content in a driver of .08\% (80mg/dL). The study period began at admission and lasted 7 days.

Results: Ninety-seven patient charts were reviewed. Average serum ethanol level on admission was $137 \mathrm{mg} / \mathrm{dL}$. Serum ethanol levels increased in 12\% of patients after administration of ethanol infusion, and levels generally decreased over time. Asymptomatic hyponatremia (serum sodium <135) occurred in $60 \%$ of patients. Benzodiazepines were administered to 52 patients (54\%); of these, only 15 received increases in infusion rates indicating appropriate protocol use. In addition, 32 of the 52 had the ethanol infusion discontinued prior Ethanol infusion for alcohol withdrawal prophylaxis in the hospitalized patient rarely induces alcohol intoxication. However, a majority of patients experience asymptomatic hyponatremia. The high rate of concomitant benzodiazepine use suggests possible low efficacy of the infusion, although low adherence to the protocol could be a contributing factor.
\end{abstract}

Key words: Clinical trial, alcohol withdrawal delirium, ethanol infusion

Corresponding author: Sharmila Dissanaike, MD Contact Information: Sharmila.dissanaike@ttuhsc.edu DOI: $10.12746 /$ swrccc2016.0416.214 


\section{INTRODUCTION}

Excessive alcohol consumption is the third leading preventable cause of death in the United States, with 2.3 million years of potential life lost in 2001. ${ }^{1}$ The lifetime prevalence of DSM-IV alcohol abuse in the general population is $17.8 \%$. These data contrast with the prevalence of alcoholism and withdrawal in trauma and surgical patients. Chronic alcoholism occurs in $50-60 \%$ of trauma patients, and alcohol misuse is as high as $43 \%$ in some surgical patients. ${ }^{2}$ The prevalence of Alcohol Withdrawal Syndrome (AWS) is correspondingly increased in these populations, with reported rates in surgical and trauma patients of $16 \%$ and $31 \%$, respectively. ${ }^{3,4}$ Additionally, predicting the occurrence of alcohol withdrawal syndrome (AWS) is difficult, as the severity of alcohol abuse does not correlate with severity of AWS. ${ }^{5}$ The initial symptoms of AWS reflect autonomic hyperactivity. However, if left untreated, AWS can progress to disorientation and seizures with possible respiratory and cardiovascular collapse. ${ }^{6}$

Multiple modes of prophylaxis for AWS exist. Benzodiazepines remain the mainstay, and several studies have shown acceptable efficacy and safety profiles. ${ }^{7-9}$ However, due to concerns that benzodiazepines may cause excessive sedation or promote delirium in patients who are often receiving simultaneous high dose opioids for analgesia and who may have concomitant head injury, clinicians have been reluctant to use aggressive benzodiazepine based AWS protocols in these patients. Surgical specialties have historically used oral alcohol instead for this purpose, with a typical prescription being "two beers with meals," for example. This approach runs counter to the notion that an acute illness or injury is a good opportunity for intervention in alcohol dependence, and oral alcohol may have side effects. However, the most significant drawback is the erratic dosing and distribution with this approach, which results in a high rate of failure. ${ }^{10}$

Our group and others have developed and implemented an intravenous ethanol infusion protocol that attempts to counteract some of the negative aspects of oral alcohol administration and have shown that ethanol infusion is a feasible option for AWS prophylaxis. ${ }^{11}$ A comparison study of ethanol, flunitrazepam-clonidine, clomethiazole-haloperidol, and flunitrazepam-haloperidol used for AWS prophylaxis in ICU patients found the failure rate of ethanol prophylaxis in preventing AWS was 4\% (2 of 50 patients). This was not significantly different from the other methods used in this study. ${ }^{7}$ Further, a standardized protocol of ethanol prophylaxis significantly reduced the rate of withdrawal symptoms from $20 \%$ to $7 \%$ and decreased the duration of treatment in surgical patients. ${ }^{10}$ However, one recurrent concern raised by many healthcare providers has been potential for intoxication from the infusion, with corresponding adverse effects. The primary aim of this study was to determine the blood ethanol levels of patients receiving an established intravenous ethanol protocol for AWS prophylaxis and to assess the frequency of intoxication. Secondary aims were to evaluate safety and efficacy of the protocol in a primarily trauma-surgical population.

\section{Methods}

Setting: This study was conducted at a Level 1 Trauma Center and American Burn Association (ABA) verified regional burn center located within an academic teaching hospital. This facility has four adult ICUs, including: Trauma/Surgical, Burn, Cardiovascular, and Medical ICUs for a total of 82 adult ICU beds and 420 adult ward beds.

Clinical Practice: Benzodiazepines, oral ethanol, and intravenous ethanol are all available and used for AWS prophylaxis at this institution, at the discretion of the treating physician team. Any patient with a known history of daily ethanol use exceeding 1 drink per day, regular episodes of binge drinking greater than two days per week, a previous history of alcohol withdrawal, or current drinking with history of treatment for alcohol related disease process is eligible for alcohol withdrawal prophylaxis. Additionally, all trauma patients are routinely screened for alcohol dependence using a standard tool as part of Level 1 Trauma Center criteria mandated by the American College of Surgeons. As a result, screening for alcohol dependence is standard practice for surgical admissions in particu- 
lar. Local practice patterns have resulted in surgical specialties primarily using the ethanol infusion while medical specialties use benzodiazepine prophylaxis only. We use a $10 \%$ ethanol solution mixed in dextrose $5 \%$ in water administered at an hourly rate of $0.4 \mathrm{ml} / \mathrm{kg} /$ hour using ideal body weight with rate adjustments outlined in the protocol as depicted in Figure 1. Briefly, for signs and symptoms of withdrawal the rate is increased; if none arise then the infusion rate is decreased and tapered off over a 96-hour period. When the protocol was first developed at our institution in 2003 , a commercial solution was available for use; however, the manufacturer discontinued it in 2006 due to the lack of a profit margin. Subsequently, the ethanol infusion has been constituted by the inpa- tient pharmacy, resulting in increased costs.

Data Collection: This was a retrospective cohort review of all patients receiving ethanol infusion from April 2008 through March 2013. Since the study was conducted primarily for quality assurance and patient safety purposes, an exemption from formal IRB review was granted. Data collected included baseline characteristics and demographics: age, sex, weight, admission diagnosis, Glasgow Coma Score, co-morbid diagnosis and clinical outcome. The rate of ethanol administration was documented daily at midnight during the 7 day study period from admission. Serum ethanol levels were recorded at 2, 12, and 24 hours

\section{Figure 1 Alcohol Withdrawal Prophylaxis Protocol}

Indication: Any patient with known history of daily ethanol use exceeding 1 - 2 drinks per day, regular episodes of binge drinking greater than two days per week, previous history of alcohol withdrawal or current drinking with history of treatment for alcohol related disease process.

1. Initiate $10 \%$ alcohol drip at $0.4 \mathrm{ml} / \mathrm{kg} / \mathrm{hr}$ using ideal body weight.

2. Measure a blood alcohol level at 6 hours, 24 hours, and 72 hours after the infusion is started. If blood alcohol level is greater than $80 \mathrm{mg} / \mathrm{dl}(0.08 \%)$, hold infusion for 2 hours and decrease rate by $50 \%$.

3. Monitor patient for signs and symptoms of alcohol withdrawal. Signs and symptoms of alcohol withdrawal include: sweating, pulse greater than $100 \mathrm{bpm}$, increased hand tremor, insomnia, nausea or vomiting, transient visual, tactile or auditory hallucinations or illusions, psychomotor agitation, anxiety, Grand mal seizures.

*Ensure symptoms are not due to general medical condition or by another mental disorder.

4. If the patient has no signs or symptoms of alcohol withdrawal after 24 hours from start, decrease the rate by $20 \%$ after 48 hours from start, decrease rate further by $50 \%$ after 72 hours from start, decrease rate further by $50 \%$ after 84 hours from start, and then discontinue infusion.

5. If patient develops signs and symptoms of alcohol withdrawal, increase rate by $50 \%$. If symptoms of alcohol withdrawal continue for 6 hours after the infusion is increased, notify the resident on call.

\section{***Do not hold or discontinue alcohol infusion for diagnostic or operative procedures***}

The alcohol infusion is appropriate for patients admitted to a floor status level of care. 
from admission for the first day, followed by levels every 24 hours until the end of the 7 day study period. Intoxication was defined using the Texas limit for blood alcohol content in a driver of $0.08 \%(80 \mathrm{mg} / \mathrm{dL})$. Benzodiazepine and anti-emetic use and indication during the study period were recorded. Serum sodium levels were reviewed. The lowest sodium level measured in each patient over the 7 day study period was recorded, and each chart was reviewed for documentation of symptomatic hyponatremia requiring treatment. Charts were reviewed for documentation of AWS and for increased ethanol infusion rates or use of anti-emetics and benzodiazepines. Patients were classified into three groups based on alcohol withdrawal symptoms: the first was apparent or documented alcohol withdrawal; the second was no sign of alcohol withdrawal; and the third was possible alcohol withdrawal based on documentation of increased ethanol infusion rates or anti-emetic and benzodiazepine use, but AWS was not listed as a diagnosis. Data collectors audited $10 \%$ of the charts picked at random to verify consistency in data abstraction. Statistical analysis was performed using Microsoft Excel.

\section{RESULTS}

Ninety-seven patient charts were reviewed. The cohort included 90 men and 7 women with an average age of 49 years. The average hospital length of stay was 7.03 days, and there were 4 deaths (4.12\%).
Ethanol infusion was started in both intensive care and floor units. Ninety-three percent of patients were managed by either surgery, trauma, or burn teams, while $7.2 \%$ of patients were managed by medical care teams. Clinical outcomes indicated that $81.4 \%$ were discharged home, $13.4 \%$ of patients were discharged to another facility, and $1 \%$ of patients were transferred. No significant changes in the average Glasgow Coma Scale (GCS) score were noted over the 7 day study period (14/15 on admission versus $14 / 15$ on discharge).

Intravenous ethanol was commonly initiated on day 1 of hospitalization; the peak infusion rate occurred on day 3 of the hospitalization. Average alcohol level on admission was $137 \mathrm{mg} / \mathrm{dL}$ with a range of 0 to $409 \mathrm{mg} / \mathrm{dL}$. Positive ethanol levels were found in the serum of $49.5 \%$ of patients after initiation of the ethanol infusion, with serum ethanol levels mostly decreasing over time. Only $12 \%$ of patients experienced an increase in alcohol levels after administration of ethanol infusion. On average, these patients' ethanol levels were $17.2 \mathrm{mg} / \mathrm{dL}$ on admission and increased $46.8 \mathrm{mg} / \mathrm{dL}$ to $64 \mathrm{mg} / \mathrm{dL}$ before beginning to trend downward. Two patients $(2 \%)$ were below the legal limit of $80 \mathrm{mg} / \mathrm{dL}$ and subsequently rose above this limit. The trend of ethanol levels over time is illustrated in Figure 2 showing a clear decrease in measured ethanol levels over time from admission. Average ethanol levels decreased every day in these patients (Table 1).

Figure 2 Alcohol level over time

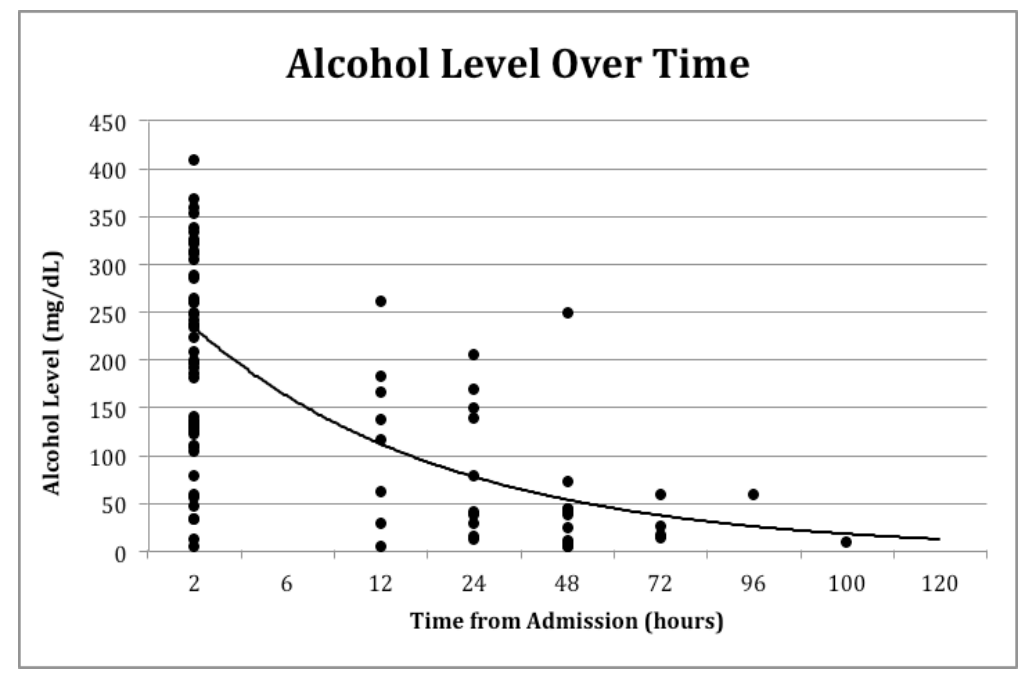


Table 1. Average serum ethanol levels over time

\begin{tabular}{|l|l|l|l|l|l|l|l|}
\hline $\begin{array}{l}\text { Hour } \\
\mathbf{0 - 2}\end{array}$ & $\begin{array}{l}\text { Hour } \\
\mathbf{6 - 1 2}\end{array}$ & $\begin{array}{l}\text { Hour } \\
\mathbf{1 3 - 2 4}\end{array}$ & $\begin{array}{l}\text { Hour 25- } \\
\mathbf{4 8}\end{array}$ & $\begin{array}{l}\text { Hour 49- } \\
\mathbf{7 2}\end{array}$ & $\begin{array}{l}\text { Hour 73- } \\
\mathbf{9 6}\end{array}$ & $\begin{array}{l}\text { Hour 97- } \\
\mathbf{1 2 0}\end{array}$ & $\begin{array}{l}\text { Hours } \\
\mathbf{1 2 1}+\end{array}$ \\
\hline $\begin{array}{l}137.1 \mathrm{mg} / \\
\mathrm{dL}\end{array}$ & $\begin{array}{l}41.8 \mathrm{mg} / \\
\mathrm{dL}\end{array}$ & $\begin{array}{l}19.6 \mathrm{mg} / \\
\mathrm{dL}\end{array}$ & $8.4 \mathrm{mg} / \mathrm{dL}$ & $4.3 \mathrm{mg} / \mathrm{dL}$ & $2.2 \mathrm{mg} / \mathrm{dL}$ & $0.7 \mathrm{mg} / \mathrm{dL}$ & $0 \mathrm{mg} / \mathrm{dL}$ \\
\hline
\end{tabular}

Infusion rate for the ethanol infusion was also evaluated. The peak ethanol infusion rate occurred on day 3 of admission. Total volume of ethanol infused per 24 hour period over the 7 day study period is shown in Figure 3. Peak average infusion total for the study period was $66 \mathrm{ml}(6.6 \mathrm{~g})$ on day 3 . For reference, one standard drink in the United States contains $14 \mathrm{~g}$ of ethanol according to the Centers for Disease Control. ${ }^{12}$ Therefore, the maximum total infusion for one day on average is less than half of a standard alcoholic beverage. period. Of these, 21 had hyponatremia on admission. The range of lowest serum sodium levels was 119$143 \mathrm{mEq} / \mathrm{L}$, with an average of $133 \mathrm{mEq} / \mathrm{L}$. There were no documented cases of symptomatic hyponatremia requiring treatment.

An antiemetic was given in $38 \%$ of patients at any time during the 7-day study period. Benzodiazepines were administered to $54 \%$ of the patients during the study period, but the indication for use was not documented in the majority of cases. Of the 52 patients who received benzodiazepines, only 15 re-

Figure 3 Average ethanol infusion per day

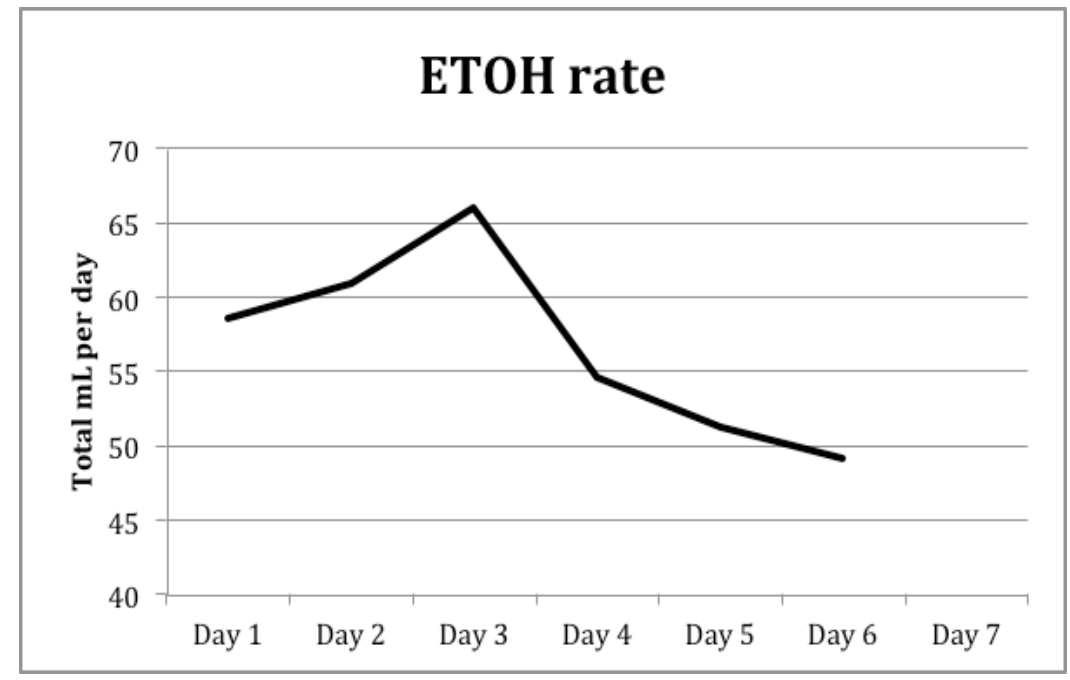

Twenty six percent of patients had documented AWS, $8 \%$ had possible AWS, and $66 \%$ had no AWS. Thirteen patients $(13.4 \%)$ received oral ethanol in addition to ethanol infusions. Of these, 9 had no AWS, and 2 had possible AWS.

Hyponatremia (serum sodium level $<135$ ) occurred in $58(60 \%)$ patients during the 7 day study ceived increases in ethanol infusion rates indicating appropriate protocol use. In addition, 32 of the 52 had the ethanol infusion discontinued early, before the scheduled protocol wean.

\section{Discussion}

Ethanol infusion was used primarily by sur- 
gical services in our hospital as opposed to medical services. The ethanol infusion seems to cause hyponatremia. However, no cases of symptomatic hyponatremia were documented in this cohort. Additionally, the infusion rarely causes intoxication; however, there was a significant rate of concomitant benzodiazepine administration, indicating a potential lower efficacy rate than previously reported by this group.

It is difficult to diagnose AWS in critically ill patients, especially in those who also have pain and delirium, which are commonly encountered in the intensive care unit. ${ }^{13}$ After a patient has been identified as having AWS or is at high risk of developing symptoms, it is prudent to implement preventative strategies to avoid more severe symptoms. ${ }^{14}$ The pharmacotherapeutic regimen should be designed to achieve three goals. The first goal is to ensure the patient is comfortable, awake, calm, and cooperative. This may not always be practical in the event of AWS, in which the patient may require large doses of sedative drugs in order to maintain control of AWS symptoms. The second goal is to prevent complications, such as severe autonomic symptoms, hallucinations, and seizures. The third goal is to reduce long term central nervous system complications that may occur due to repeated withdrawal episodes. To achieve these goals, the recommended first line therapy for both prevention and management of AWS are benzodiazepines due to their ability to increase transmission of GABA. However, the use of benzodiazepines may lead to excessive sedation and respiratory depression. Consequently, less sedating alternative therapies would potentially be very useful. ${ }^{13}$

Ethanol is a small polar molecule that has both lipophilic and hydrophilic characteristics. The amphipathic abilities of ethanol help explain its pharmacokinetics within the body. Ethanol's lipophilic characteristics result in passive diffusion across the cell membranes. The combination of the hydrophilic and polar properties of the ethanol molecule makes it completely water-soluble with a similar volume of distribution to total body water. ${ }^{13}$ These characteristics of the ethanol molecule indicate that its pharmacology is complex and why it is described as the "perfect drug". ${ }^{15}$
Therefore, intravenous ethanol should, in theory, be an effective alternative means of preventing and treating AWS, while causing less sedation, which would allow trauma patients to better participate in respiratory maneuvers to limit atelectasis and decrease secretions and in other aspects of patient care. ${ }^{14}$ However, critics argue that intravenous ethanol has a short duration of action, a narrow margin of safety, and possible precipitation of acute hepatic failure. In addition, the use of ethanol in AWS contradicts the prevention efforts of alcohol abuse. 10,14 Many of these conclusions are made on the assumption that to detoxify a patient the dose required for intravenous ethanol is initially equal to the patient's average daily consumption. However, the amount of intravenous ethanol to prevent withdrawal may be much less. In fact, when administered at very small doses, it has been successful in aiding in detoxification, as well as preventing acute manifestations of AWS. ${ }^{16}$ Furthermore, using intravenous ethanol does not provide the main addictive reinforcements such as intoxication and socialization, which are characteristics of oral alcohol use, and therefore does not represent a barrier to efforts to start rehabilitation from alcohol dependency. ${ }^{10}$

Ethanol has been shown to be as effective as other methods of AWS prophylaxis. Spies et al conducted a prospective randomized control trial and found that prophylaxis with ethanol, benzodiazepines, chlormethiazole, and haloperidol all had similar efficacy in preventing AWS. Further, the ICU stay did not differ among the groups. ${ }^{7}$ In a systematic review of randomized control trials, Unger et al concluded that either benzodiazepines or ethanol can be used for AWS prophylaxis in ICU patients based upon similar efficacy. ${ }^{9}$ Additionally, our group has shown that a standardized protocol for ethanol prophylaxis is significantly more effective in avoiding AWS than nonstandard ethanol administration. ${ }^{10}$

Unfortunately, in the current study we did note a significant failure rate with ethanol infusions, with $8 \%$ developing AWS and an additional 26\% having signs or symptoms of possible AWS. Of equal concern was the high proportion of patients who were administered benzodiazepines while receiving the etha- 
nol infusion; in the absence of clearly documented indications for the benzodiazepine administration, we have to assume that a significant proportion of these patients may have received the drug to treat AWS. It is likely that this occurred due to the assumption on the part of the provider that any signs or symptoms represent failure of the protocol and the need to switch to alternate medication. However, even benzodiazepine protocols are commonly administered on an "as needed/ PRN" basis rather than a regimen of scheduled medications in AWS prophylaxis protocols. ${ }^{17}$ To consider the development of any signs or symptoms of withdrawal as a failure of the protocol is a higher bar than expected in standard practice. The significant limitation of this study was that there was no systematic validated method of measuring and documenting AWS in use during this study, thus preventing us from accurately assessing efficacy of the protocol. This is unfortunately a widespread problem in many busy trauma centers, although increased awareness of the importance of AWS and the high rate of recidivism in injured patients with alcohol dependency have led to nationwide efforts to improve the monitoring and treatment of alcohol related complications in these patients. ${ }^{18} \mathrm{~A}$ protocol based on the CIWA-Ar scale has since been included in the electronic order set for AWS prophylaxis at our institution. However, during the time period for this study there was inconsistent use of formalized protocols for monitoring of AWS.

\section{LIMITATIONS}

Limitations of this study include the small sample size and the retrospective nature of the research. The inability to determine the indications for benzodiazepine administration in this cohort is also a significant confounding factor in judging the overall efficacy of the protocol, beyond the documented $8 \%$ failure rate. Inconsistent use and documentation of formalized instruments to monitor AWS also led to deficits in the documentation available for review.

\section{Conclusions}

Ethanol infusion for alcohol withdrawal prophylaxis in the hospitalized patient does not induce alcohol intoxication. However, a significant rate of deviations from the protocol and concomitant use of benzodiazepines was noted, raising doubts about the efficacy of the protocol. Additionally, asymptomatic hyponatremia was observed in a majority of patients. More research is needed into comparing efficacy of ethanol infusion with other modes of AWS prophylaxis.

\section{REFERENCES}

1. Centers for Disease Control and Prevention (CDC). Alcohol-attributable deaths and years of potential life lost-United States, 2001. MMWR Morb Mortal Wkly Rep 2004; 53(37):866-870.

2. Spies CD, Dubisz N, Neumann T, et al. Therapy of alcohol withdrawal syndrome in intensive care unit patients following trauma: Results of a prospective, randomized trial. Crit Care Med 1996; 24(3):414-422.

3. Spies CD, Neuner B, Neumann T, et al. Intercurrent complications in chronic alcoholic men admitted to the intensive care unit following trauma. Intensive Care Med. 1996; 22(4):286-293.

4. de Wit M, Best AM, Gennings C, Burnham EL, Moss M. Alcohol use disorders increase the risk for mechanical ventilation in medical patients. Alcohol Clin Exp Res 2007; 31(7):1224-1230.

5. Goodson CM, Clark BJ, Douglas IS. Predictors of severe alcohol withdrawal syndrome: A systematic review and meta-analysis. Alcohol Clin Exp Res 2014; 38(10):2664-2677. 6. Hall W, Zador D. The alcohol withdrawal syndrome. Lancet 1997; 349(9069):1897-1900. 
7. Spies CD, Dubisz N, Funk W, et al. Prophylaxis of alcohol withdrawal syndrome in alcohol-dependent patients admitted to the intensive care unit after tumour resection. $\mathrm{Br}$ J Anaesth 1995; 75(6):734-739.

8. Mayo-Smith MF. Pharmacological management of alcohol withdrawal. A meta-analysis and evidence-based practice guideline. American Society of Addiction Medicine working group on pharmacological management of alcohol withdrawal. JAMA 1997; 278(2):144-151.

9. Ungur LA, Neuner B, John S, Wernecke K, Spies C. Prevention and therapy of alcohol withdrawal on intensive care units: Systematic review of controlled trials. Alcohol Clin Exp Res 2013; 37(4):675-686.

10. Dissanaike S, Halldorsson A, Frezza EE, Griswold J. An ethanol protocol to prevent alcohol withdrawal syndrome. J Am Coll Surg 2006; 203(2):186-191.

11. Awissi DK, Lebrun G, Coursin DB, Riker RR, Skrobik Y. Alcohol withdrawal and delirium tremens in the critically ill: A systematic review and commentary. Intensive Care Med 2013; 39(1):16-30.

12. Zakhari S. Overview: How is alcohol metabolized by the body? Alcohol Res Health 2006; 29(4):245-254.

13. Hodges B, Mazur JE. Intravenous ethanol for the treatment of alcohol withdrawal syndrome in critically ill patients. Pharmacotherapy 2004; 24(11):1578-1585.

14. Weinberg JA, Magnotti LJ, Fischer PE, et al. Comparison of intravenous ethanol versus diazepam for alcohol withdrawal prophylaxis in the trauma ICU: Results of a randomized trial. J Trauma 2008; 64(1):99-104.

15. Kent W. The pharmacokinetics of alcohol in healthy adults. . 2012.

16. Hansbrough JF, Zapata-Sirvent RL, Carroll WJ, Johnson R, Saunders CE, Barton CA. Administration of intravenous alcohol for prevention of withdrawal in alcoholic burn patients. Am J Surg 1984; 148(2):266-269.

17. Maldonado JR, Nguyen LH, Schader EM, Brooks JO, 3rd. Benzodiazepine loading versus symptom-triggered treatment of alcohol withdrawal: A prospective, randomized clinical trial. Gen Hosp Psychiatry 2012; a center staff. J Trauma 1999; 47(6):1131-5; discussion 1135-9. 